\title{
LINEAR OPENNESS OF MULTIFUNCTIONS IN METRIC SPACES
}

\author{
CORNELIU URSESCU
}

Received 9 January 2004 and in revised form 25 October 2004

We discuss linear openness results for multifunctions with closed graphs in metric spaces.

\section{Introduction}

Let $X$ and $Y$ be metric spaces, let $F: X \rightarrow Y$ be a multifunction, and recall the elementary definitions of openness and almost openness of $F$ at a point $(x, y) \in \operatorname{graph}(F)$. The multifunction $F$ is said to be open at $(x, y)$ if for every $\epsilon>0$, there exists $\delta>0$ such that

$$
B(y, \delta) \subseteq F(B(x, \epsilon)) .
$$

Here, $B(c, r)$ stands for the open ball with center $c$ and radius $r$. The multifunction $F$ is said to be almost open at $(x, y)$ if for every $\epsilon>0$, there exists $\delta>0$ such that

$$
B(y, \delta) \subseteq \overline{F(B(x, \epsilon))} .
$$

Here, $\bar{S}$ stands for the closure of the set $S$. Various openness and almost openness notions are build on the inclusions (1.1) and (1.2). Every openness property implies the corresponding almost openness property, for $S \subseteq \bar{S}$. It is expected for the converse implication to be true in case that the multifunction $F$ has a closed graph. In this regard, we recall the result in [12, Chapter 6, Lemma 36, page 202] (see also [7, Lemma 6.4.1, page 435]), where $Y$ is a more general space, namely, a uniform space. The metric version of this result revolves around the composite inclusion

$$
\overline{F\left(B\left(x, \epsilon^{\prime}\right)\right)} \subseteq F(B(x, \epsilon)) .
$$

Theorem 1.1. Let the metric space $X$ be complete. Let the multifunction $F$ have a closed graph. Suppose that for every $\epsilon>0$, there exists $\delta>0$ such that for every $(x, y) \in \operatorname{graph}(F)$, there holds the inclusion (1.2). Then for every $\epsilon>0$, for every $\epsilon^{\prime} \in(0, \epsilon)$, and for every $x \in \operatorname{domain}(F)$, there holds the inclusion (1.3). Consequently, for every $\epsilon>0$, there exists $\delta>0$ such that for every $(x, y) \in \operatorname{graph}(F)$, there holds the inclusion (1.1). 
Our aim is to enlarge on Theorem 1.1 in case that the "nonlinear" openness inclusion (1.1) is replaced with the "linear" openness inclusion

$$
B(y, \omega \epsilon) \subseteq F(B(x, \epsilon)),
$$

whereas the "nonlinear" almost openness inclusion (1.2) is replaced with the "linear" almost openness inclusion

$$
B(y, \omega \epsilon) \subseteq \overline{F(B(x, \epsilon))} .
$$

Here, $\omega>0$ is a real number. The three main results are stated in Section 2. The second and third results involve a class of special metric spaces. The proofs of the main results are given in Section 3. A few counterexamples are given in Section 5. Section 6 contains characterizations of the class of special metric spaces.

\section{Main results}

The first linear openness result states the equivalence of the strongest openness condition based on the inclusion (1.4) to the corresponding almost openness condition. It is a straightforward corollary of Theorem 1.1.

Theorem 2.1. Let the metric space $X$ be complete. Let the multifunction $F$ have a closed graph. Let $\omega>0$ be a real number. The following two conditions are equivalent:

(i) for every $(x, y) \in \operatorname{graph}(F)$ and for every $\epsilon>0$, there holds the inclusion (1.4);

(ii) for every $(x, y) \in \operatorname{graph}(F)$ and for every $\epsilon>0$, there holds the inclusion (1.5).

The proof of Theorem 2.1 is given in Section 3. Theorem 2.1 may fail if the metric space $X$ is not complete. A counterexample is given in Section 5 .

A better linear openness result states the equivalence of the same openness condition to a weaker almost openness condition. It is also a corollary of Theorem 1.1. It can be derived in case that $Y$ is a particular metric space. If $y \in Y, \delta>0$, and $\delta^{\prime}>0$, then

$$
B\left(B(y, \delta), \delta^{\prime}\right) \subseteq B\left(y, \delta+\delta^{\prime}\right)
$$

in case that $Y$ is a general metric space. Here, $B(C, r)$ stands for the union of all $B(c, r)$ with $c \in C$. Moreover,

$$
B\left(B(y, \delta), \delta^{\prime}\right)=B\left(y, \delta+\delta^{\prime}\right)
$$

in case that $Y$ is a normed space endowed with the metric generated by the norm.

Definition 2.2. The metric space $Y$ is said to resemble normed spaces if for every $y \in Y$, for every $\delta>0$, and for every $\delta^{\prime}>0$, there holds the equality (2.2).

Equivalently, $Y$ resembles normed spaces if and only if $B(y, \delta) \cap B\left(y^{\prime}, \delta^{\prime}\right) \neq \varnothing$ whenever $\delta+\delta^{\prime}>d\left(y, y^{\prime}\right)$. Accordingly, $\overline{B(y, \delta)}=B(y, \delta) \cup S(y, \delta)$. Here, $S(y, \delta)$ stands for the sphere with center $y$ and radius $\delta$ (cf. [4, page 20]). Two characterizations of metric spaces $Y$ which resemble normed spaces are given in Section 6. 
Theorem 2.3. Let the metric space $X$ be complete and let the metric space $Y$ resemble normed spaces. Let the multifunction $F$ have a closed graph. Let $\omega>0$ be a real number. The following two conditions are equivalent:

(i) for every $(x, y) \in \operatorname{graph}(F)$ and for every $\epsilon>0$, there holds the inclusion (1.4);

(ii) for every $\zeta>0$ there exists $\epsilon \in(0, \zeta)$, such that for every $(x, y) \in \operatorname{graph}(F)$, there holds the inclusion (1.5).

The proof of Theorem 2.3 is given in Section 3. Theorem 2.3 may fail if the metric space $Y$ does not resemble normed spaces. A counterexample is given in Section 5.

The best linear openness result states the equivalence of the same openness condition to the weakest almost openness condition based on the inclusion (1.5). It is no longer a corollary of Theorem 1.1. It can be derived in case that $Y$ is a complete metric space which resembles normed spaces.

Theorem 2.4. Let the metric space $X$ be complete, and let the metric space $Y$ be complete and resemble normed spaces. Let the multifunction $F$ have a closed graph. Let $\omega>0$ be a real number. The following two conditions are equivalent:

(i) for every $(x, y) \in \operatorname{graph}(F)$ and for every $\epsilon>0$, there holds the inclusion (1.4);

(ii) for every $(x, y) \in \operatorname{graph}(F)$ and for every $\zeta>0$, there exists $\epsilon \in(0, \zeta)$ such that there holds the inclusion (1.5).

The proof of Theorem 2.4 is given in Section 4. Theorem 2.4 may fail if either the metric space $Y$ does not resemble normed spaces or it is not complete. Counterexamples are given in Section 5 .

The three almost openness conditions and the common openness condition of Theorems 2.1, 2.3, and 2.4 appear in the elementary flowchart of implications

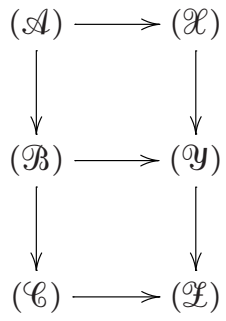

which involves the openness conditions

$(A)$ for every $(x, y) \in \operatorname{graph}(F)$ and for every $\epsilon>0$, there holds the inclusion (1.4);

$(\mathscr{B})$ for every $\zeta>0$, there exists $\epsilon \in(0, \zeta)$ such that for every $(x, y) \in \operatorname{graph}(F)$, there holds the inclusion (1.4);

$(\mathscr{C})$ for every $(x, y) \in \operatorname{graph} F$ and for every $\zeta>0$, there exists $\epsilon \in(0, \zeta)$ such that there holds the inclusion (1.4);

as well as the corresponding almost openness conditions

$(\mathscr{X})$ for every $(x, y) \in \operatorname{graph}(F)$ and for every $\epsilon>0$, there holds the inclusion (1.5);

(y) for every $\zeta>0$, there exists $\epsilon \in(0, \zeta)$ such that for every $(x, y) \in \operatorname{graph}(F)$, there holds the inclusion (1.5); 


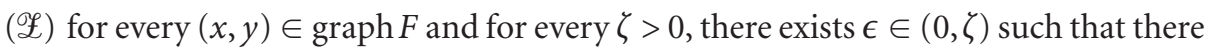
holds the inclusion (1.5).

Theorem 2.1 adds the expected implication $(\mathscr{X}) \rightarrow(\mathscr{A})$ to the flowchart above, Theorem 2.3 adds the better implication $(\mathscr{Y}) \rightarrow(\mathscr{A})$, and Theorem 2.4 adds the best implication $(\mathscr{Z}) \rightarrow(\mathscr{A})$.

\section{Proof of Theorems 2.1 and 2.3}

To prove Theorem 2.1, we have to derive some properties of the moduli of uniform openness and uniform almost openness of $F$, denoted by $\Delta$ and $\bar{\Delta}$, respectively. For every $\epsilon>0$, the extended real number $\Delta(\epsilon)$ equals the supremum of all $\delta>0$ such that the inclusion (1.1) holds for all $(x, y) \in \operatorname{graph}(F)$, whereas the extended real number $\bar{\Delta}(\epsilon)$ equals the supremum of all $\delta>0$ such that the inclusion (1.2) holds for all $(x, y) \in \operatorname{graph}(F)$. By convention, $\sup \varnothing=0$. Obviously the extended real functions $\Delta$ and $\bar{\Delta}$ are nondecreasing, $0 \leq \Delta(\epsilon) \leq \bar{\Delta}(\epsilon)$,

$$
\begin{aligned}
& B(y, \Delta(\epsilon)) \subseteq F(B(x, \epsilon)), \\
& B(y, \bar{\Delta}(\epsilon)) \subseteq \overline{F(B(x, \epsilon))} .
\end{aligned}
$$

By convention, $B(y, 0)=\varnothing$ and $B(y,+\infty)=Y$. The following result is a quantitative corollary of Theorem 1.1 (cf. [15, Theorem 2.1, page 505]).

Lemma 3.1. Let the metric space $X$ be complete. Let the multifunction $F$ have a closed graph. Suppose that $\bar{\Delta}(\epsilon)>0$ for all $\epsilon>0$. Then $\Delta(\epsilon) \geq \lim _{\epsilon^{\prime} \uparrow \epsilon} \bar{\Delta}\left(\epsilon^{\prime}\right)$ for all $\epsilon>0$. Consequently, $\Delta(\epsilon)>0$ for all $\epsilon>0$.

Proof of Theorem 2.1. The almost openness condition of Theorem 2.1 states that $\bar{\Delta}(\epsilon) \geq$ $\omega \epsilon$ for all $\epsilon>0$. According to Lemma 3.1, $\Delta(\epsilon) \geq \omega \epsilon$ for all $\epsilon>0$, and the openness condition of Theorem 2.1 follows.

To prove Theorem 2.3, we have to derive further properties of the moduli $\Delta$ and $\bar{\Delta}$. An extended real function $\Gamma:(0,+\infty) \rightarrow[0,+\infty]$ is said to be superadditive if $\Gamma\left(\epsilon+\epsilon^{\prime}\right) \geq$ $\Gamma(\epsilon)+\Gamma\left(\epsilon^{\prime}\right)$ for all $\epsilon>0$ and for all $\epsilon^{\prime}>0$. In this case (cf. [10, pages 131, 135]),

$$
\inf _{\epsilon>0} \frac{\Gamma(\epsilon)}{\epsilon}=\lim _{\epsilon \downarrow 0} \frac{\Gamma(\epsilon)}{\epsilon} .
$$

Indeed, if $\epsilon>0$ and $\epsilon^{\prime}>0$, then there exists a unique integer $\iota$ such that $\iota \epsilon^{\prime} \leq \epsilon<(\iota+$ 1) $\epsilon^{\prime}$, hence $\Gamma(\epsilon) / \epsilon \geq\left(\iota \Gamma\left(\epsilon^{\prime}\right)\right) /\left((\iota+1) \epsilon^{\prime}\right)$. Since $\lim _{\epsilon^{\prime} \downarrow 0} \iota=+\infty$, it follows $\Gamma(\epsilon) / \epsilon \geq$ limsup $\operatorname{\epsilon u}_{\epsilon^{\prime} \downarrow} \Gamma\left(\epsilon^{\prime}\right) / \epsilon^{\prime}$, and the desired equality follows.

Lemma 3.2. Let the metric space $Y$ resemble normed spaces. Then the moduli $\Delta$ and $\bar{\Delta}$ are superadditive.

Proof. Let $\epsilon>0$ and $\epsilon^{\prime}>0$. We have to show that $\bar{\Delta}\left(\epsilon+\epsilon^{\prime}\right) \geq \bar{\Delta}(\epsilon)+\bar{\Delta}\left(\epsilon^{\prime}\right)$. The inequality is obvious if either $\bar{\Delta}(\epsilon)=0$ or $\bar{\Delta}\left(\epsilon^{\prime}\right)=0$. Suppose that both $\bar{\Delta}(\epsilon)>0$ and $\bar{\Delta}\left(\epsilon^{\prime}\right)>0$, and 
let $\delta \in(0, \bar{\Delta}(\epsilon))$ and $\delta^{\prime} \in\left(0, \bar{\Delta}\left(\epsilon^{\prime}\right)\right)$. Then

$$
B\left(B(y, \delta), \delta^{\prime}\right) \subseteq B\left(\overline{F(B(x, \epsilon))}, \delta^{\prime}\right)=B\left(F(B(x, \epsilon)), \delta^{\prime}\right) \subseteq \overline{F\left(B\left(B(x, \epsilon), \epsilon^{\prime}\right)\right)},
$$

because $B(S, r)=B(\bar{S}, r)$ whenever $r>0$. Since $Y$ resembles normed spaces, it follows $B\left(y, \delta+\delta^{\prime}\right) \subseteq \overline{F\left(B\left(x, \epsilon+\epsilon^{\prime}\right)\right)}$, hence $\delta+\delta^{\prime} \leq \bar{\Delta}\left(\epsilon+\epsilon^{\prime}\right)$, and the desired inequality follows. The fact that $\Delta$ is superadditive can be derived in the same way.

Lemma 3.2 may fail if the metric space $Y$ does not resemble normed spaces. A counterexample is given in Section 5.

Proof of Theorem 2.3. The almost openness condition of Theorem 2.3 states that for every $\zeta>0$, there exists $\epsilon \in(0, \zeta)$ such that $\bar{\Delta}(\epsilon) \geq \omega \epsilon$, hence $\limsup _{\epsilon \downarrow 0} \bar{\Delta}(\epsilon) / \epsilon \geq \omega$. According to Lemma 3.2, $\bar{\Delta}$ is superadditive, hence $\inf _{\epsilon>0} \bar{\Delta}(\epsilon) / \epsilon \geq \omega$. According to Lemma 3.1, $\Delta(\epsilon) \geq \omega \epsilon$ for all $\epsilon>0$, and the openness condition of Theorem 2.3 follows.

\section{Proof of Theorem 2.4}

In contrast with Theorems 2.1 and 2.3, the almost openness condition of Theorem 2.4 does not state anything about the modulus $\bar{\Delta}$, so Lemma 3.1 cannot be applied.

Since the condition $\mathscr{A}$ always implies the condition $\mathscr{L}$ (recall the elementary flowchart at the end of Section 2), Theorem 2.4 is a straightforward corollary of Lemma 4.1 and Theorem 4.2 below. Lemma 4.1 prefigures a new condition, which is based on the rather elaborate relation

$$
\varnothing \neq B(v, d(v, y)-\theta \omega \epsilon) \cap B(y, \omega \epsilon) \cap F(B(x, \epsilon)),
$$

and which is weaker than the condition $\mathscr{E}$ provided that $Y$ resembles normed spaces. Theorem 4.2 states the equivalence of the condition $\mathscr{A}$ to the new condition no matter whether $Y$ resembles normed spaces.

Lemma 4.1. Let the metric space $Y$ resemble normed spaces. Let $\omega>0$ be a real number. Let $(x, y) \in \operatorname{graph}(F)$. Suppose that for every $\zeta>0$, there exists $\epsilon \in(0, \zeta)$ such that there holds the inclusion (1.5). Then for every $v \in Y \backslash\{y\}$ and for every $\theta \in(0,1)$, there exists $\epsilon \in(0, d(v, y) /(\theta \omega))$ such that there holds the relation (4.1).

Proof. Let $v \in Y \backslash\{y\}$, let $\theta \in(0,1)$, and denote $\zeta=d(v, y) /(\theta \omega)$. By hypothesis, there exists $\epsilon \in(0, \zeta)$ such that the relation (1.5) holds. Since $Y$ resembles normed spaces, it follows that the intersection of the open balls $B(v, d(v, y)-\theta \omega \epsilon)$ and $B(y, \omega \epsilon)$ is nonempty. Since this intersection is a nonempty open subset of $\overline{F(B(x, \epsilon))}$, it follows that the relation (4.1) holds.

Theorem 4.2. Let the metric spaces $X$ and $Y$ be complete. Let the multifunction $F$ have a closed graph. Let $\omega>0$ be a real number. The following two conditions are equivalent:

(i) for every $(x, y) \in \operatorname{graph}(F)$ and for every $\epsilon>0$, there holds the inclusion (1.4);

(ii) for every $(x, y) \in \operatorname{graph}(F)$, for every $v \in Y \backslash\{y\}$, and for every $\theta \in(0,1)$, there exists $\epsilon \in(0, d(v, y) /(\theta \omega))$ such that there holds the relation (4.1). 
Theorem 4.2 is a straightforward corollary of Lemmas 4.3 and 4.4 below. The obvious Lemma 4.3 prefigures the $v$-components of the condition $\mathscr{A}$ through the simple relation

$$
v \in F(B(x, \epsilon)) .
$$

Lemma 4.4 states the equivalence of every $v$-component of the condition $\mathscr{A}$ to the corresponding $v$-component of the new condition.

Lemma 4.3. Let $\omega>0$ be a real number. Let $(x, y) \in \operatorname{graph}(F)$. The following two conditions are equivalent:

(i) for every $\epsilon>0$, there holds the inclusion (1.4);

(ii) for every $v \in Y \backslash\{y\}$ and for every $\epsilon>d(v, y) / \omega$, there holds the relation (4.2).

Lemma 4.4. Let the metric spaces $X$ and $Y$ be complete. Let the multifunction $F$ have a closed graph. Let $\omega>0$ be a real number. Let $v \in Y$. The following two conditions are equivalent:

(i) for every $(x, y) \in \operatorname{graph}(F)$ such that $y \neq v$ and for every $\epsilon>d(v, y) / \omega$, there holds the relation (4.2);

(ii) for every $(x, y) \in \operatorname{graph}(F)$ such that $y \neq v$ and for every $\theta \in(0,1)$, there exists $\epsilon \in$ $(0, d(v, y) /(\theta \omega))$ such that there holds the relation (4.1).

Proof. First, let the former condition be satisfied, let $(x, y) \in \operatorname{graph}(F)$ such that $y \neq v$, let $\theta \in(0,1)$, and let $\epsilon \in(d(y, v) / \omega, d(y, v) /(\theta \omega))$. Since (4.2) holds, it follows that $v$ belongs to the right-hand side of (4.1), and the latter condition is satisfied too.

Finally, let the latter condition be satisfied, let $(x, y) \in \operatorname{graph}(F)$ such that $y \neq v$, and let $\epsilon>d(v, y) / \omega$. We have to prove that (4.2) holds. Let $\theta \in(d(v, y) /(\omega \epsilon), 1)$. Following the spirit of some ideas in [2, page 195], [3, page 76], [11, page 572], and [13, page 30] (cf. also [16, page 222], [17, pages 81,82$]$, and [18, page 404]), we endow the space $X \times Y$ with the metric

$$
d((p, q),(a, b))=\max \{\omega d(p, a), d(q, b)\}
$$

and we apply the variational principle of Ekeland [8, page 324] to the function

$$
(p, q) \in \operatorname{graph}(F) \longrightarrow d(v, q) \in \mathbb{R}
$$

in order to get a point $(a, b) \in \operatorname{graph}(F)$ such that

$$
d(v, b)+\theta \cdot d((a, b),(x, y)) \leq d(v, y)
$$

(see [2, page 195] and [14, page 815]);

$$
d(v, b)<d(v, q)+\theta \cdot d((p, q),(a, b))
$$


for every $(p, q) \in \operatorname{graph}(F) \backslash\{(a, b)\}$. According to the former inequality of the Ekeland principle, $d((a, b),(x, y))<\omega \epsilon$, so $a \in B(x, \epsilon)$. Note that $b \in F(a) \subseteq F(B(x, \epsilon))$. We claim that $v=b$. Suppose, to the contrary, that $b \neq v$. By hypothesis, there exists $\alpha \in$ $(0, d(v, b) /(\theta \omega))$ such that the set

$$
S=B(v, d(v, b)-\theta \omega \alpha) \cap B(b, \omega \alpha) \cap F(B(a, \alpha))
$$

is nonempty. Let $q \in S$. Since $q \in F(B(a, \alpha))$, it follows that there exists $p \in B(a, \alpha)$ such that $q \in F(p)$. Since $d(q, b)<\omega \alpha$, it follows that $d((p, q),(a, b))<\omega \alpha$. Since $d(q, v)<$ $d(v, b)-\theta \omega \alpha$, it follows $q \neq b$. Further, it follows from the latter inequality of the Ekeland principle that $d(v, b)<(d(v, b)-\theta \omega \alpha)+\theta \cdot \omega \alpha$, therefore $0<0$, a contradiction. To conclude, $v=b$, and the proof is accomplished.

Lemma 4.4 may fail if $F$ does not have a closed graph. A counterexample is given in Section 5 .

\section{Counterexamples}

Our first counterexample shows that if the metric space $X$ is not complete, then Theorems $2.1,2.3$, and 2.4 may fail, namely, the almost openness condition $\mathscr{X}$ does not imply the openness condition $\mathscr{A}$ (recall again the elementary flowchart at the end of Section 2 ).

Counterexample 5.1. Let $\mathbb{Q}$ be the metric space of rational numbers and let $\mathbb{R}$ be the metric space of real numbers. Let $F: \mathbb{Q} \rightarrow \mathbb{R}$ be the multifunction given by $\operatorname{graph}(F)=$ $\{(x, y) ; x=y\}$. Let $\omega=1$.

Then $\mathbb{Q}$ is not complete, whereas $\mathbb{R}$ is complete and resembles normed spaces, and $F$ has a closed graph. On the one hand, if $(x, y) \in \operatorname{graph}(F)$ and $\epsilon>0$, then $F\left(B_{\mathbb{Q}}(x, \epsilon)\right)=$ $B_{\mathbb{R}}(y, \epsilon) \cap \mathbb{Q}$, hence $\Delta(\epsilon)=0$. Here, $B_{\mathbb{Q}}$ introduces the balls in $\mathbb{Q}$, whereas $B_{\mathbb{R}}$ introduces the balls in $\mathbb{R}$. On the other hand, $B_{\mathbb{R}}(y, \epsilon) \subseteq \overline{F\left(B_{\mathbb{Q}}(x, \epsilon)\right)}$, hence $\bar{\Delta}(\epsilon)=\epsilon$.

Our next counterexample shows that if the metric space $Y$ does not resemble normed spaces, then Theorems 2.3 and 2.4 may fail, namely, the almost openness condition 9 does not imply the openness condition $\mathscr{A}$. Neither does the following openness condition, which is implied by $\mathscr{A}$ and which implies $\mathscr{B}$ :

(i) there exists $\zeta>0$ such that for every $\epsilon \in(0, \zeta)$ and for every $(x, y) \in \operatorname{graph}(F)$, there holds the inclusion (1.4).

The counterexample shows also that Lemma 3.2 may fail, that is, the moduli $\Delta$ and $\bar{\Delta}$ are not superadditive.

Counterexample 5.2. Let $Y$ be a set with at least two points and let the set $Y$ be endowed with the discrete metric (see [4, page 30]),

$$
d(p, q)= \begin{cases}0 & \text { if } p=q \\ 1 & \text { if } p \neq q\end{cases}
$$

Let $F: Y \rightarrow Y$ be the multifunction given by $\operatorname{graph}(F)=\{(x, y) ; x=y\}$. Let $\omega=2$. 
Then $Y$ is complete and

$$
B(y, \delta)= \begin{cases}\{y\} & \text { if } \delta \leq 1 \\ Y & \text { if } \delta>1\end{cases}
$$

Since

$$
B(B(y, 1), 1)=\{y\} \subset Y=B(y, 2)
$$

it follows that $Y$ does not resemble normed spaces. Further, the multifunction $F$ has a closed graph and $B(y, \epsilon)=F(B(x, \epsilon))=\overline{F(B(x, \epsilon))}$ for all $(x, y) \in \operatorname{graph}(F)$ and for all $\epsilon>0$, hence

$$
\bar{\Delta}(\epsilon)=\Delta(\epsilon)= \begin{cases}1 & \text { if } 0<\epsilon \leq 1 \\ +\infty & \text { if } 1<\epsilon\end{cases}
$$

Since $\Delta(1 / 2)+\Delta(1 / 2)>\Delta(1)$, it follows the modulus $\Delta$ is not superadditive. Finally, if $\epsilon \in$ $(0,1 / 2]$ and $(x, y) \in \operatorname{graph}(F)$, then $B(y, 2 \epsilon) \subseteq B(y, 1)=\{y\} \subseteq F(B(x, \epsilon))$, but if $(x, y) \in$ $\operatorname{graph}(F)$ and $\epsilon \in(1 / 2,1]$, then $B(y, 2 \epsilon)=Y \nsubseteq\{y\}=F(B(x, \epsilon))$.

Our next counterexample shows that if the metric space $Y$ is not complete, then Theorem 2.4 may fail, namely, the almost openness condition $\mathscr{E}$ does not imply the openness condition $\mathscr{A}$. Neither does the following openness condition, which is implied by $\mathscr{A}$ and which implies $\mathscr{b}$ :

(i) for every $(x, y) \in \operatorname{graph}(F)$, there exists $\zeta>0$ such that for every $\epsilon \in(0, \zeta)$, there holds the inclusion (1.4).

Counterexample 5.3. Let $\mathbb{R}$ be the metric space of real numbers and let $\mathbb{Q}$ be the metric space of rational numbers. Let $F: \mathbb{R} \rightarrow \mathbb{Q}$ be the multifunction given by $\operatorname{graph}(F)=$ $\{(x, y) ; x \in(-\sqrt{2},+\sqrt{2}), x=y\}$. Let $\omega=1$.

Then $\mathbb{R}$ is complete, $\mathbb{Q}$ is not complete but resembles normed spaces, and $F$ has a closed graph. On the one hand, if $(x, y) \in \operatorname{graph}(F)$ and $\epsilon>0$, then $F\left(B_{\mathbb{R}}(x, \epsilon)\right)=$ $B_{\mathbb{Q}}(y, \epsilon) \cap(-\sqrt{2},+\sqrt{2})$, hence $B_{\mathbb{Q}}(y, \epsilon)=F\left(B_{\mathbb{R}}(x, \epsilon)\right)$ for all $(x, y) \in \operatorname{graph}(F)$ and for all $\epsilon \in(0, \sqrt{2}-|x|)$. On the other hand, $\operatorname{range}(F) \neq \mathbb{Q}$.

Our final counterexample shows that Lemma 4.4 may fail if the multifunction $F$ does not have a closed graph.

Counterexample 5.4. Let $\mathbb{R}$ be the metric space of real numbers. Let $F: \mathbb{R} \rightarrow \mathbb{R}$ be the multifunction given by $\operatorname{graph}(F)=\{(x, y) ; x>0, y=x\}$. Let $\omega=1$. Let $v=0$.

Then $\mathbb{R}$ is complete, but $F$ does not have a closed graph. Note also that, if $(x, y) \in$ $\operatorname{graph}(F)$ and $\epsilon>0$, then $F(B(x, \epsilon))=B(y, \epsilon) \cap(0,+\infty)$. On the one hand, if $(x, y) \epsilon$ $\operatorname{graph}(F), y \neq v, \theta \in(0,1)$, and $\epsilon=d(y, v)$, then $\epsilon \in(0, d(y, v) / \theta)$ and the right-hand side of the relation (4.1) equals the nonempty set $B(v, d(y, v)-\theta \epsilon) \cap B(y, \epsilon)$. On the other hand, $v \notin \operatorname{range}(F)$. 


\section{Metric spaces resembling normed spaces}

Every normed space endowed with the metric induced by the norm resembles normed spaces. So does every convex subset of a normed space, but this may be false for nonconvex subsets. Let $Y$ be the unit circle centered at the origin of the Hilbert space $\mathbb{R}^{2}$. On the one hand, if $Y$ is endowed with the metric induced by the chord length, $d\left(y, y^{\prime}\right)=$ $\left\|y-y^{\prime}\right\|$, then $Y$ does not enjoy the resembling property. For example, if $y \in Y$, then

$$
B_{Y}\left(B_{Y}(y, \sqrt{2}), \sqrt{2}\right)=Y \backslash\{-y\} \subset Y=B_{Y}(y, 2 \sqrt{2}) .
$$

Here, $B_{Y}$ introduces the balls in $Y$. On the other hand, if $Y$ is endowed with the metric induced by the minimum arc length, $d\left(y, y^{\prime}\right)=2 \arcsin \left(\left\|y-y^{\prime}\right\| / 2\right)$, then $Y$ enjoys the resembling property. Resuming the preceding example, if $y \in Y$, then

$$
B_{Y}\left(B_{Y}\left(y, \frac{\pi}{2}\right), \frac{\pi}{2}\right)=Y \backslash\{-y\}=B_{Y}(y, \pi)
$$

Note that the two metrics on $Y$ generate the same topology, so the resembling notion cannot be completely characterized by the topology.

Next we give two characterizations of metric spaces $Y$, which resemble normed spaces. One characterization makes use of chains in $Y$, that is, finite sequences $\chi=\left\{\chi_{0}, \chi_{1}, \ldots, \chi_{n}\right\}$ of points of $Y$. If $\chi$ is a chain in $Y$, we define the mesh of the chain through

$$
\operatorname{mesh}(\chi)=\max \left\{d\left(\chi_{0}, \chi_{1}\right), \ldots, d\left(\chi_{n-1}, \chi_{n}\right)\right\}
$$

and we define the length of the chain through

$$
\operatorname{length}(\chi)=d\left(\chi_{0}, \chi_{1}\right)+\cdots+d\left(\chi_{n-1}, \chi_{n}\right)
$$

Another characterization makes use of functions $\psi: D \rightarrow Y$, where

$$
D=\left\{\frac{i}{2^{n}} ; n \in\{0,1, \ldots\}, i \in\left\{0,1, \ldots, 2^{n}\right\}\right\}
$$

stands for the set of all dyadic numbers. If $\lambda \geq 0$ is a real number, we say that a function $\psi: D \rightarrow Y$ is $\lambda$-Lipschitzian if

$$
d(\psi(t), \psi(s)) \leq \lambda|t-s|
$$

for all $s \in D$ and for all $t \in D$. In case that the metric space $Y$ is complete, every $\lambda$ Lipschitzian function $\psi: D \rightarrow Y$ can be uniquely extended to a $\lambda$-Lipschitzian function $\bar{\psi}:[0,1] \rightarrow Y$ through the equality

$$
\operatorname{graph}(\bar{\psi})=\overline{\operatorname{graph}(\psi)}
$$


Theorem 6.1. Let $Y$ be a metric space. The following three conditions are equivalent to each other:

(1) the metric space $Y$ resembles normed spaces;

(2) for every $y \in Y$, for every $y^{\prime} \in Y$, for every $\mu>0$, and for every $\lambda>d\left(y, y^{\prime}\right)$, there exists a chain $\chi$ in $Y$ such that length $(\chi) \leq \lambda, \operatorname{mesh}(\chi) \leq \mu$, as well as $\chi_{0}=y$ and $\chi_{n}=y^{\prime}$;

(3) for every $y \in Y$, for every $y^{\prime} \in Y$, and for every $\lambda>d\left(y, y^{\prime}\right)$, there exists a $\lambda$ Lipschitzian function $\psi: D \rightarrow Y$ such that $\psi(0)=y$ and $\psi(1)=y^{\prime}$.

Proof. First, let condition (3) be satisfied. In order to show that condition (2) is satisfied too, let $y \in Y, y^{\prime} \in Y, \lambda>d\left(y, y^{\prime}\right)$, and $\mu>0$. There exists $n \in \mathbb{N}$ such that $\lambda / 2^{n} \leq \mu$. Consider a function $\psi$ provided by condition (3), and let $\chi$ be the chain given by $\chi_{i}=$ $\psi\left(i / 2^{n}\right)$, where $i \in\left\{0,1, \ldots, 2^{n}\right\}$. Then $\chi_{0}=y$ and $\chi_{2^{n}}=y^{\prime}, \operatorname{length}(\chi) \leq \lambda$, and $\operatorname{mesh}(\chi) \leq$ $\lambda / 2^{n}$, which proves that condition (2) is satisfied too.

Further, let the condition (2) be satisfied. In order to show that the condition (1) is satisfied too, let $y \in Y, y^{\prime} \in Y, \delta>0$, and $\delta^{\prime}>0$ such that $d\left(y, y^{\prime}\right)<\delta+\delta^{\prime}$. We must show that $B(y, \delta) \cap B\left(y^{\prime}, \delta^{\prime}\right)$ is nonempty. There exist $\lambda>d\left(y, y^{\prime}\right)$ and $\mu>0$ such that $\lambda+\mu<\delta+\delta^{\prime}$. Consider a chain $\chi$ provided by the condition (2), and let $i$ be the greatest one such that $d\left(\chi_{0}, \chi_{i}\right)<\delta$. We assert that $d\left(\chi_{i}, \chi_{n}\right)<\delta^{\prime}$, hence $\chi_{i}$ belongs to both $B(y, \delta)$ and $B\left(y^{\prime}, \delta^{\prime}\right)$. The assertion is obvious if $i=n$. If $i<n$, then $\delta \leq d\left(\chi_{0}, \chi_{i+1}\right) \leq d\left(\chi_{0}, \chi_{i}\right)+$ $\operatorname{mesh}(\chi)$. Since $d\left(\chi_{0}, \chi_{i}\right)+d\left(\chi_{i}, \chi_{n}\right) \leq \operatorname{length}(\chi)$, it follows that $d\left(\chi_{i}, \chi_{n}\right) \leq \operatorname{length}(\chi)-$ $d\left(\chi_{0}, \chi_{i}\right) \leq$ length $(\chi)+\operatorname{mesh}(\chi)-\delta \leq \lambda+\mu-\delta<\delta^{\prime}$, our assertion is justified, and condition (1) is satisfied too.

Finally, let condition (1) be satisfied. In order to show that condition (3) is satisfied too, let $y \in Y, y^{\prime} \in Y$, and $\lambda>d\left(y, y^{\prime}\right)$. We can suppose $y \neq y^{\prime}$. Recall that $D=\cup_{n \geq 0} D_{n}$, where $D_{n}=\left\{i / 2^{n} ; i \in\left\{0, \ldots, 2^{n}\right\}\right\}$, and denote by $\Psi_{n}$ the set of all functions $\psi: D_{n} \rightarrow Y$ such that $\psi(0)=y$, such that $\psi(1)=y^{\prime}$, and such that

$$
0<d(\psi(t), \psi(s))<\lambda|t-s|
$$

for every two successive points $s \in D_{n}$ and $t \in D_{n}$. First, we note that there exists a unique $\psi_{0} \in \Psi_{0}$. Further, we note that for every $n \geq 0$ and for every $\psi_{n} \in \Psi_{n}$, there exists $\psi_{n+1} \in$ $\Psi_{n+1}$ such that $\psi_{n+1}$ is an extension of $\psi_{n}$. To define $\psi_{n+1}$ on $D_{n+1} \backslash D_{n}$, let $\theta \in D_{n+1} \backslash D_{n}$. Then there exist two successive points $s \in D_{n}$ and $t \in D_{n}$ such that $\theta=(s+t) / 2$. Consider $r=\min \left\{d\left(\psi_{n}(t), \psi_{n}(s)\right), \lambda|t-s| / 2\right\}$ and observe that the set

$$
B\left(\psi_{n}(s), r\right) \cap B\left(\psi_{n}(t), r\right)
$$

is nonempty because $Y$ resembles normed spaces and $2 r>d\left(\psi_{n}(t), \psi_{n}(s)\right)$. Consider a point $q$ of this nonempty set. Since $r \leq d\left(\psi_{n}(t), \psi_{n}(s)\right)$, it follows that $q \neq \psi_{n}(s)$ and $q \neq$ $\psi_{n}(t)$. Since $r \leq \lambda|t-s| / 2$, it follows that

$$
\begin{aligned}
& d\left(q, \psi_{n}(s)\right)<\lambda|\theta-s| \\
& d\left(\psi_{n}(t), q\right)<\lambda|t-\theta| .
\end{aligned}
$$

Now, define $\psi_{n+1}(\theta)=q$. Then $\psi_{n+1} \in \Psi_{n+1}$. 


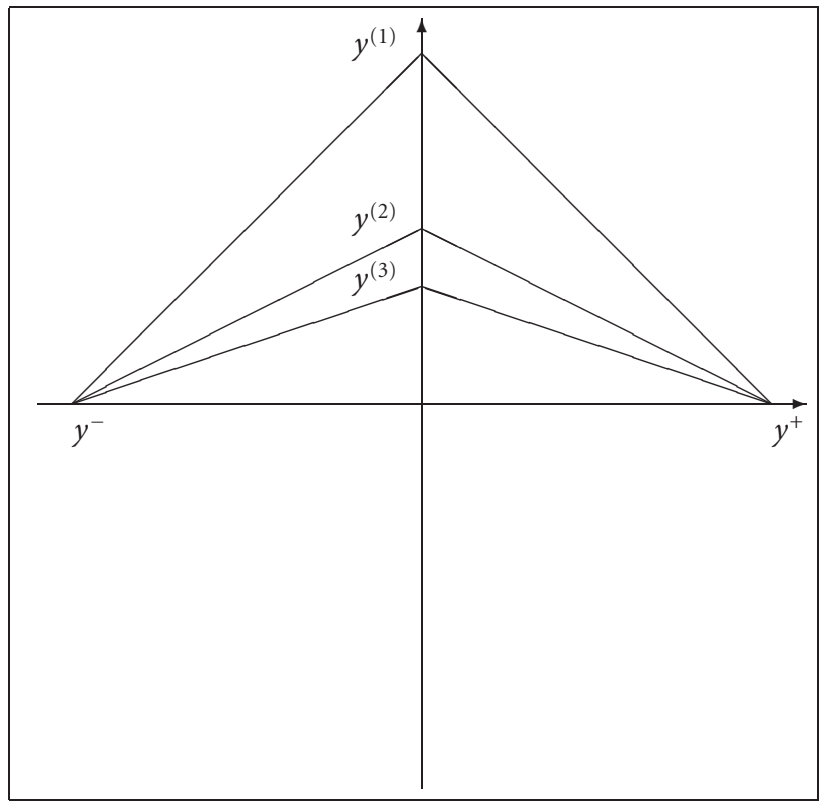

Figure 6.1. The subsets $Y^{(1)}, Y^{(2)}$, and $Y^{(3)}$ of the set $Y$.

To conclude, there exists a unique function $\psi: D \rightarrow Y$ such that $\psi$ is an extension of every $\psi_{n}$, hence $\psi(0)=y$ and $\psi(1)=y^{\prime}$. Moreover, if $s \in D$ and $t \in D$, then there exists $n$ such that $s \in D_{n}$ and $t \in D_{n}$. Since $d\left(\psi_{n}(\tau), \psi_{n}(\sigma)\right)<\lambda|\tau-\sigma|$ for every pair of successive points $\tau$ and $\sigma$ of $D_{n}$, it follows $d\left(\psi_{n}(t), \psi_{n}(s)\right)<\lambda|t-s|$. Therefore, $\psi$ is $\lambda$-Lipschitzian, condition (3) is satisfied too, and the proof of the theorem is accomplished.

Theorem 6.1(2) and (3) can be rephrased by using established metric terms. For example, the $\lambda$-free variant of the condition (2) states that the metric space $Y$ is well chained (see [19, page 13]), whereas the $\lambda$-component states that the metric $d$ is intrinsic (see [1, pages 9,10$]$ and $[6$, page 77$])$.

Further, if the metric space $Y$ is complete, then condition (3) states that $Y$ is connected by rectifiable curves and the distance between two points equals the infimum of the lengths of the rectifiable curves connecting those points. If $Y$ is finitely compact, which means that the bounded, closed subsets of $Y$ are compact (see [5, pages 6, 403]), then there exist connecting curves of shortest lengths (see [5, page 25]). If $Y$ is not finitely compact, the existing property may fail. In this regard, we will construct an appropriate subset $Y$ in the Hilbert space $\mathbb{R}^{2}$. First, we consider, in $\mathbb{R}^{2}$, the two points $y^{ \pm}=( \pm 1,0)$ and the sequence of points $y^{(k)}=(1 / k, 0)$. Finally, we consider the set $Y=\cup_{k} Y^{(k)}$, where each $Y^{(k)}$ consists of the two line segments joining $y^{(k)}$ to $y^{ \pm}$(see Figure 6.1; cf. [9, Figure 4, page 239]).

On the one hand, if $d_{Y}\left(y, y^{\prime}\right)=\left\|y-y^{\prime}\right\|$, then $Y$ is not a complete metric space and it does not resemble normed spaces. On the other hand, if $d_{Y}\left(y, y^{\prime}\right)$ equals the infimum of the lengths of rectifiable curves in $Y$ joining $y$ to $y^{\prime}$, then $Y$ is a complete metric 
space which resembles normed spaces, but $d_{Y}\left(y^{-}, y^{+}\right)$does not equal the minimum of the lengths of rectifiable curves in $Y$ joining $y^{-}$to $y^{+}$. Indeed, every such a curve contains a set $Y^{(k)}$, of which length, $2 \sqrt{1+1 / k^{2}}$, exceeds 2 , whereas $d_{Y}\left(y_{-}, y_{+}\right)=2$.

\section{References}

[1] A. D. Aleksandrov, Vnutrennyaya Geometriya Vypuklyh Poverhnostě̆ [Intrinsic Geometry of Convex Surfaces], OGIZ, Moscow, 1948.

[2] J.-P. Aubin and A. Cellina, Differential Inclusions, Grundlehren der Mathematischen Wissenschaften, vol. 264, Springer-Verlag, Berlin, 1984.

[3] J.-P. Aubin and H. Frankowska, On inverse function theorems for set-valued maps, J. Math. Pures Appl. (9) 66 (1987), no. 1, 71-89.

[4] N. Bourbaki, Éléments de Mathématique. Topologie Générale, Actualités Sci. Ind., no. 1045, Hermann et Cie., Paris, 1948.

[5] H. Busemann, The Geometry of Geodesics, Academic Press, New York, 1955.

[6] Convex Surfaces, Interscience Tracts in Pure and Applied Mathematics, no. 6, Interscience Publishers, New York, 1958.

[7] R. E. Edwards, Functional Analysis. Theory and Applications, Holt, Rinehart and Winston, New York, 1965.

[8] I. Ekeland, On the variational principle, J. Math. Anal. Appl. 47 (1974), 324-353.

[9] F. Hausdorff, Grundzüge der Mengenlehre, Veit \& Comp., Leipzig, 1914.

[10] E. Hille, Functional Analysis and Semi-Groups, American Mathematical Society Colloquium Publications, vol. 31, American Mathematical Society, New York, 1948.

[11] A. D. Ioffe, On the local surjection property, Nonlinear Anal. 11 (1987), no. 5, 565-592.

[12] J. L. Kelley, General Topology, D. Van Nostrand, New York, 1955.

[13] J.-P. Penot, Open mappings theorems and linearization stability, Numer. Funct. Anal. Optim. 8 (1985), no. 1-2, 21-35.

[14] The drop theorem, the petal theorem and Ekeland's variational principle, Nonlinear Anal. 10 (1986), no. 9, 813-822.

[15] V. Pták, A quantitative refinement of the closed graph theorem, Czechoslovak Math. J. 24(99) (1974), 503-506.

[16] C. Ursescu, Tangency and openness of multifunctions in Banach spaces, An. Ştiinţ. Univ. Al. I. Cuza Iaşi Secţ. I a Mat. 34 (1988), no. 3, 221-226.

[17] _ Multifunction openness and set tangency, Mem. Secț. Ştiinţ. Acad. Română Ser. IV 17 (1994), 79-85.

[18] Inherited openness, Rev. Roumaine Math. Pures Appl. 41 (1996), no. 5-6, 401-416.

[19] G. T. Whyburn, Analytic Topology, American Mathematical Society Colloquium Publications, vol. 28, American Mathematical Society, New York, 1942.

Corneliu Ursescu: Institute of Mathematics “O. Mayer,” Romanian Academy Iaşi Branch, 700505 Iaşi, Romania

E-mail address: uc@academie.is.edu.ro 


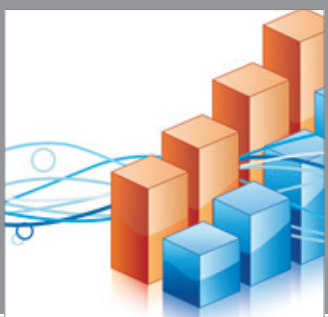

Advances in

Operations Research

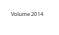

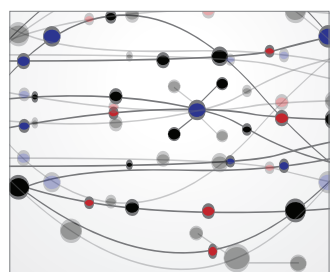

\section{The Scientific} World Journal
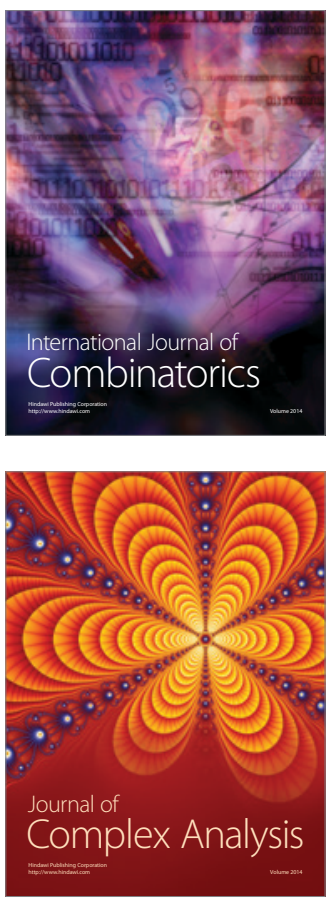

International Journal of

Mathematics and

Mathematical

Sciences
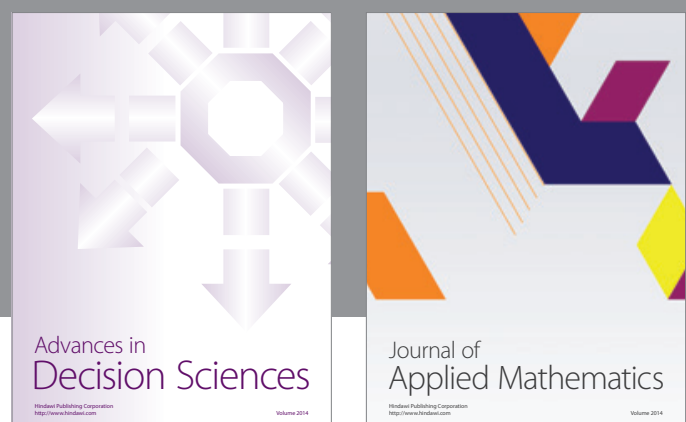

Journal of

Applied Mathematics
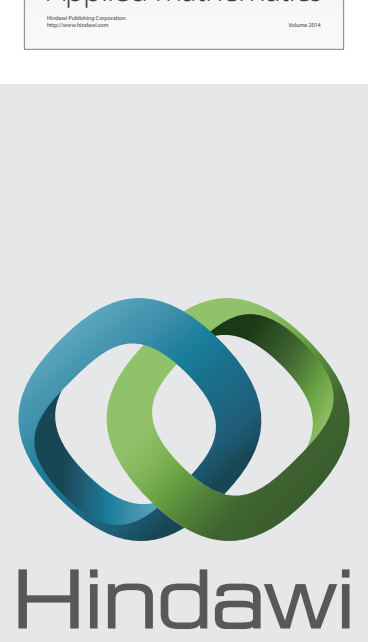

Submit your manuscripts at http://www.hindawi.com
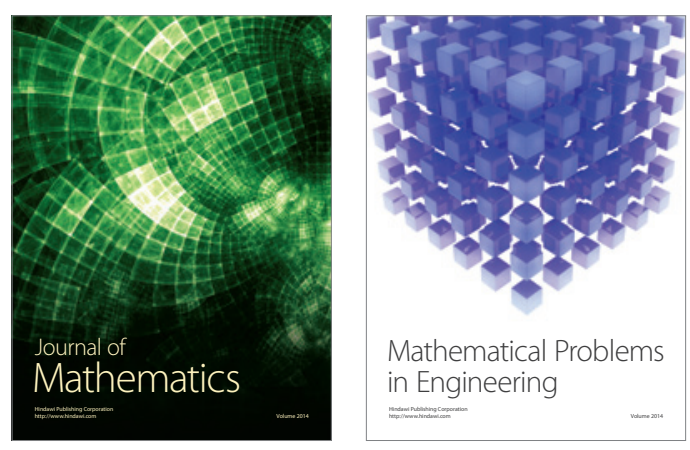

Mathematical Problems in Engineering
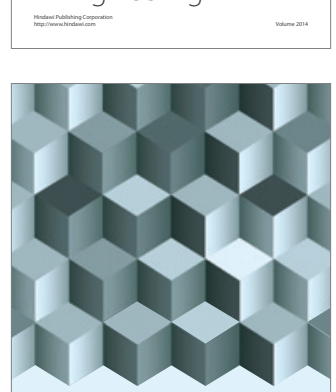

Journal of

Function Spaces
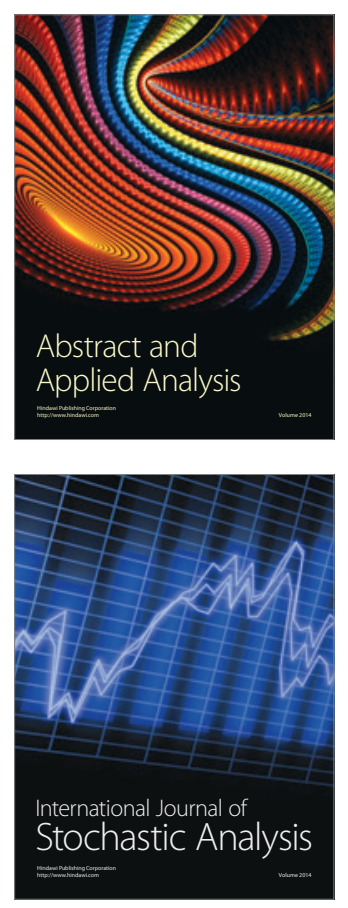

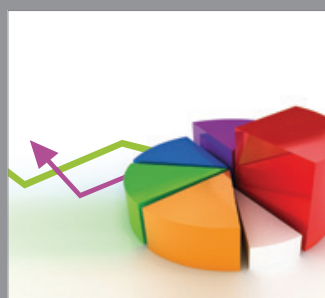

ournal of

Probability and Statistics

Promensencen
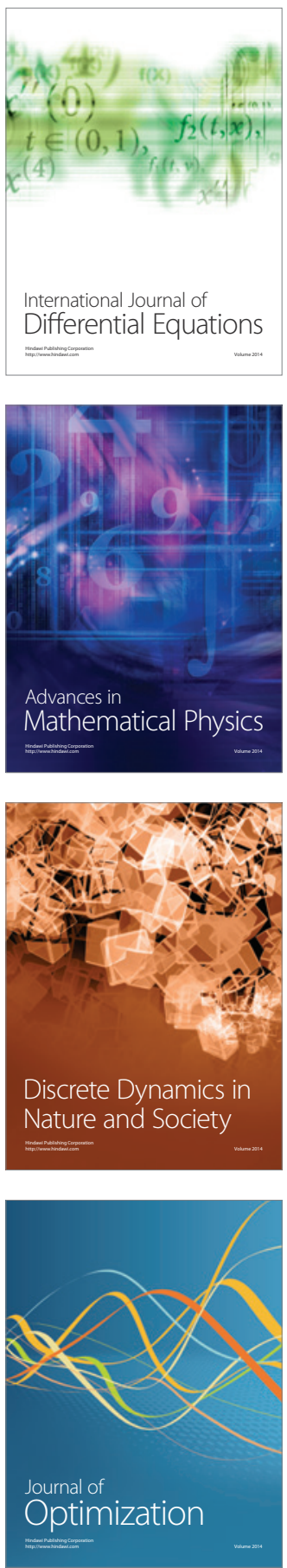